\title{
Critical Items to Consider before Adopting Advocacy of Digital Subtraction Angiography
}

Kenneth D. Candido, MD

$\mathrm{n}$ the present issue of Pain Physician, Dr. Nader and associates have described their results of a meta-analysis of studies assessing the ability of digital subtraction angiography (DSA) to more reliably identify vascular penetration, compared with real-time fluoroscopy, during epidural steroid injections (ESIs).

The authors identified 49 reports and 4 manuscripts relating to 1,290 ESIs with 188 intravascular events; 148 events identified by fluoroscopy, with DSA detecting an additional 40 events missed by fluoroscopy, for a $32 \%$ improvement rate of detection. They also note a $30 \%$ "missed-events" rate for detecting vascular penetration when fluoroscopy is used, and aptly point out that this does not translate to an increased incidence of complications, as no single patient in their review sustained a neurological insult, whether or not DSA or fluoroscopy was used.

Among their conclusions was the statement, "This discrepancy suggests that factors other than vascular events also play a role in complications." They nonetheless advocate for an increased use of DSA over real-time fluoroscopy, while noting the 2.3 - 4.3-fold increase in radiation exposure, per case, when DSA is used compared to the use of fluoroscopy. In 3 of the 4 studies they cite, examination included procedures performed at sacral levels; and in the same number of studies, Quincke needles (sharp, cutting bevels) were used for transforaminal injections.

There are several critical things that we need to reconcile before adopting the advocacy of the present authors for increasing the use of DSA while performing ESIs, whether by a transforaminal route or an interlaminar one.

The most glaring deficiency of adopting the suggestion to increase use of DSA relates to the fallibility of DSA to identify arterial injection of contrast medium versus venous injection. Neurologic injury follows arterial, and not venous, injection of nonsoluble (i.e., "particulate") steroids. It has not been reliably reported following venous injection of nonsoluble or soluble (i.e., "nonparticulate") agents, or following arterial injection of soluble steroids (i.e., dexamethasone). The present paper does nothing to enhance an argument in favor of DSA as a radiological adjunct that more competently identifies the more sinister arterial vascular injection, compared with the more benign venous vascular injection, particularly in light of the acknowledged greater exposure to ionizing radiation consistently reported with DSA use.

In a case report wherein DSA and fluoroscopy were both used to confirm suspected vascular injection, both modalities were effective in identifying the spread of contrast medium in a vascular pattern spreading towards the central neuraxis, hence consistent with an arterial injection, leading to the decision to abort the procedure (1). Another case report seemed to show how DSA identified an arterial spread of contrast medium, which was originally missed using fluoroscopy (2), again leading to aborting the injection; however, as was appropriately pointed out in a subsequently written letter to the editor evaluating the original needle placement for a left C6 nerve root injection, the needle which led to a vascular penetration in the first place had not been competently placed into the neuroforamen using standard International Spine Intervention Society needle placement guidelines (3).

McClean et al (4), in a retrospective chart review of 134 patients, compared real-time fluoroscopy versus DSA in 177 cervical transforaminal epidural steroid injections (TFESI). Intravascular injection was detected in $18 \%$ of cervical TFESI using real-time fluoroscopy versus $32.8 \%$ using DSA ( $P=$ 0.0471 ). However, all the vascular angiograms identified by BOTH live fluoroscopy as well as by DSA were noted to be

From: Department of Anesthesiology, Advocate Illinois Masonic Medical Center, University of Illinois College of Medicine, Chicago, IL 
venous in origin. Lee et al (5) performed a prospective study of vascular flow detection rates during lumbosacral TFESI with 60 lumbar and 27 sacral foraminal injections (authors' reference 9). DSA identified 20 cases of intravascular injection (9 lumbar; 11 sacral) versus 12 identified using real-time fluoroscopy. The authors conceded that "the majority of these vascular injections were venous" (5). A closer look at this study shows that the sacral (S-1) injections were associated with a significantly higher proportion $(40.7 \%)$ of vascular uptake when compared to lumbar injections (15\%). Due to the rich venous sacral plexus, this finding is what is expected anatomically. El Abd et al (6) evaluated DSA use for vascular identification that was not found using other safety precautions such as aspiration, looking for a blood "flash" at the hub, live fluoroscopy, or a test-dose of local anesthetic (authors' reference 7). They enrolled 150 consecutive patients and performed 222 TFESI in the cervical (18.47\%), lumbar (50.9\%) and sacral levels (30.36\%). Quincke needles were used for all injections. Live fluoroscopy with contrast medium identified 46 intravascular flow patterns; DSA found an additional 5 not seen on fluoroscopy. All 5 found using DSA that were in addition to the fluoroscopically identified injections were venous injections and not arterial. This represented $2.25 \%$ of all injections performed (6). Finally, Kim et al (7) completed a large prospective study comparing fluoroscopy with DSA for identification of intravascular flow patterns (authors' reference 8). They studied 732 injections performed on 348 patients; all injections being again performed with a Quincke-type needle. Fluoroscopy found $8.1 \%$ (59 cases) while DSA found $10.5 \%$ (77 cases) of intravascular injection; $3.9 \%$ (fluoroscopy) and 6\% (DSA) were lumbar TFESI versus $22.6 \%$ (37 cases-fluoroscopy) and $26.2 \%$ (43 cases-DSA) were sacral. They actually found no statistically significant differences in identification of intravascular injection between the 2 techniques (DSA versus fluoroscopy). There was a higher likelihood of this event occurring in the elderly or during sacral injections. Once again, no distinction whatsoever was made by the authors regarding whether or not they had identified an arterial or a venous injection.

The advertised advantage of DSA is its potential to identify vascular uptake of contrast medium prior to injecting an analgesic agent, whether that be a soluble or insoluble corticosteroid. However, and unfortunately, DSA falls short of meeting that expectation because it has not been shown, by study or meta-analysis, to competently distinguish between an arterial versus a venous injection, and hence, in alerting clinicians to the potentially devastating and permanent complications associated with use of insoluble steroids injected transforaminally. The present meta-analysis fails to differentiate these respective types (venous versus arterial) of vascular injections, and hence the utility of the information, regrettably, is limited.

In conclusion, while the present paper by Nader et al adds to our appreciation that we need to continue to seek enhanced methods of safely providing interventional therapy to our patients suffering from radicular pain associated with degenerated intervertebral discs, I respectfully suggest that we take 2 steps back, both away from the fluoroscopy machine, and from embracing an increased use of the more expensive, and more radiation-exposing DSA. DSA is not, in my opinion, the answer to our safety concerns; its use will incur additional costs, both physical and economic, that our system may not be able to bear; and will bring upon us a potential false sense of security, particularly when we fail to factor human error into the interventional pain management procedure equation (8).

\section{References}

1. Baker R, Dreyfuss, P, Mercer S, Bogduk N. Cervical transforaminal injection of corticosteroids into a radicular artery: A possible mechanism for spinal cord injury. Pain 2003; 103:211-215.

2. Verrills, P, Nowesenitz G, Barnard A. Penetration of a cervical radicular artery during a transforaminal epidural injection. Pain Medicine 2010; 11:229-231.

3. Landers M: Comments on: Penetration of a cervical radicular artery during a transforaminal epidural injection. Pain Medicine 2010; 11:1306-1307.

4. McClean J, Sigler J, Plastaras C, Garvan
C, Rittenberg J. The rate of detection of intravascular injection in cervical transforaminal epidural steroid injections with and without digital subtraction angiography. PM \& $R$ 2009; 1: 636-642.

5. Lee M, Yang K, Kim Y, Jung H, Lim S, Moon D. Accuracy of live fluoroscopy to detect intravascular injection during lumbar transforaminal epidural injections. Korean J Pain 2010; 23: 18-23.

6. El Abd O, Amadera J, Pimentel D, Pimentel T. Intravascular flow detection during transforaminal epidural injec- tions: A prospective assessment. Pain Physician 2014; 17: 21-27.

7. Kim Y, Park H, Moon D. Rates of lumbosacral transforaminal injections interpreted as intravascular: Fluoroscopy alone or with digital subtraction. Anaesthesia 2013; 68:1120-1123.

8. Chang-Chien G, Candido K, Knezevic N. Digital subtraction angiography does not reliably prevent paraplegia associated with lumbar transforaminal epidural steroid injections. Pain Physician 2012; 15:515-523. 